\title{
Properties of third-order nonlinear delay dynamic equations with positive and negative coefficients
}

\author{
Xianyong Huang ${ }^{1}$ and Xun-Huan Deng ${ }^{2 *}$
}

\section{"Correspondence:}

deng_xunhuan@163.com

${ }^{2}$ Department of Mathematics,

College of Medical Information

Engineering, Guangdong

Pharmaceutical University,

Guangzhou, P.R. China

Full list of author information is

available at the end of the article

\section{Springer}

\begin{abstract}
We study asymptotic properties of a class of third-order nonlinear delay dynamic equations with positive and negative coefficients on time scales. In this paper, we establish new results on asymptotic behavior of nonoscillatory and oscillatory solutions for the corresponding equations by making a comparison with suitable first-order dynamic equations. Finally, we offer an example to illustrate the feasibility of our results.

MSC: $34 \mathrm{~N} 05 ; 34 \mathrm{C} 10$

Keywords: Nonlinear delay dynamic equations; Comparison method; Positive and negative coefficients; Oscillation; Asymptotic behavior
\end{abstract}

\section{Introduction}

We are interested in studying oscillation and nonoscillation of third-order nonlinear delay dynamic equations with positive and negative coefficients

$$
\left[b(t)\left(a(t) x^{\Delta}(t)\right)^{\Delta}\right]^{\Delta}+p(t) f(x(\tau(t)))-q(t) h(x(\delta(t)))=0, \quad t \in \mathbb{T}, t \geq t_{0} .
$$

Throughout this paper, we impose the following conditions:

(A1) $\mathbb{T}$ is an arbitrary time scale with $\sup \mathbb{T}=+\infty, b, a, p, q \in C_{r d}\left(\mathbb{T}, \mathbb{R}^{+}\right)$;

(A2) $\int_{t_{0}}^{\infty} \frac{1}{b(s)} \Delta s=\int_{t_{0}}^{\infty} \frac{1}{a(s)} \Delta s=\infty$;

(A3) $f, h: \mathbb{R} \rightarrow \mathbb{R}$ are continuous functions with $u f(u)>0$, $u h(u)>0$ for $u \neq 0$;

(A4) $\tau, \delta: \mathbb{T} \rightarrow \mathbb{T}$ are nondecreasing, $\tau(t) \leq t, \delta(t) \geq t, \lim _{t \rightarrow \infty} \tau(t)=\infty$.

Definition 1.1 A function $x$ is said to be a solution of (1.1) if a nontrivial real-valued function $x \in C_{r d}\left(\left[t_{0}, \infty\right)_{\mathbb{T}}, \mathbb{R}\right)$ with $a x^{\Delta} \in C_{r d}^{1}\left(\left[t_{0}, \infty\right)_{\mathbb{T}}, \mathbb{R}\right)$ and $b\left(a x^{\Delta}\right)^{\Delta} \in C_{r d}^{1}\left(\left[t_{0}, \infty\right)_{\mathbb{T}}, \mathbb{R}\right)$, and such that $(1.1)$ is satisfied on the interval $\left[t_{0}, \infty\right)_{\mathbb{T}}$.

About the existence and uniqueness of solutions to delay dynamic equations on time scales, we refer to [16]. As usual, a solution of (1.1) is called nonoscillatory if it is either eventually negative or eventually positive; otherwise it is called oscillatory. Equation (1.1) is said to be oscillatory provided that every solution of (1.1) is oscillatory.

(c) The Author(s) 2019. This article is distributed under the terms of the Creative Commons Attribution 4.0 International License (http://creativecommons.org/licenses/by/4.0/), which permits unrestricted use, distribution, and reproduction in any medium, provided you give appropriate credit to the original author(s) and the source, provide a link to the Creative Commons license, and indicate if changes were made. 
The theory of time scales introduced by Hilger (see [15]) unifies continuous and discrete analysis. Oscillations of delay dynamic equations are common in applications, for example, in economics, where the demand depends on current price and the supply depends on the price at an earlier time, and in the study of population dynamic models (see [3]). Compared to dynamic equations with positive coefficients, the research on oscillation and nonoscillation of dynamic equations with positive and negative coefficients has received less attention. What is more, most of the papers focus on the oscillation of second-order dynamic equations with positive and negative coefficients (see [5, 6, 8, 17, 19-21]), much fewer do on third-order dynamic equations with positive and negative coefficients. However, for third-order delay dynamic equations with positive coefficients, the oscillation theory has been developed well on the binomial equations. For example, when $q(t)=0$, $\tau(t)=t,(1.1)$ becomes the nonlinear dynamic equation

$$
\left[b(t)\left(a(t) x^{\Delta}(t)\right)^{\Delta}\right]^{\Delta}+p(t) f(x(t))=0, \quad t \in \mathbb{T}, t \geq t_{0} .
$$

If $q(t)=0, b(t)=a(t)=1, \tau(t)=t, f(x(t))=x(t)$, then (1.1) is simplified to the linear dynamic equation

$$
x^{\Delta \Delta \Delta}(t)+p(t) x(t)=0, \quad t \in \mathbb{T}, t \geq t_{0} .
$$

And it is easy to see that (1.1) can be turned into the third-order nonlinear delay dynamic equation with positive coefficients

$$
\left[b(t)\left(a(t) x^{\Delta}(t)\right)^{\Delta}\right]^{\Delta}+p(t) f(x(\tau(t)))=0, \quad t \in \mathbb{T}, t \geq t_{0} .
$$

In 2005, Erbe et al. [10] considered (1.2). With the help of the generalized Riccati transformation techniques, they established oscillation criteria which ensure that every solution of (1.2) is oscillatory or converges to zero. In 2006 and 2007, Erbe et al. [11, 12] studied (1.3), and in [12] they obtained Hille and Nehari type oscillation criteria. In 2011, Han et al. [14] extended and improved the results of [10-12]. Han et al. offered new oscillatory criteria of (1.4) and demonstrated that every solution of (1.4) oscillates or is convergent to zero.

However, when using the Riccati transformation, we meet an unexpected obstacle: the nonoscillatory solution space of (1.1) is unclear from Lemma 2.1. Agarwal et al. [1] studied oscillation of second-order dynamic equations and turned to the nonexistence of eventually positive solutions of a first-order dynamic inequality. Grace et al. [13] and Erbe et al. [9] investigated the oscillation of higher order dynamic equations to make a comparison with second-order dynamic equations and dynamic inequalities respectively. Jozef et al. [7] also used the comparison method to study the oscillatory properties of (1.1) when $\mathbb{T}=\mathbb{R}$, and then, in 2017, they [2] continued the investigation to study nonoscillation properties of (1.1).

Motivated by the works $[1,2,7,9,13]$, we obtain some sufficient conditions which guarantee that Equation (1.1) is oscillatory, and we give certain new results for asymptotic behavior of nonoscillatory solutions of (1.1). Our work generalizes and improves the main results of $[2,7]$ and those related research works in the literature for oscillation of thirdorder nonlinear delay dynamic equations. Moreover, our results are new for the corresponding third-order nonlinear difference equations and differential equations. 
For convenience, we denote

$$
A(t)=\int_{t_{1}}^{t} \frac{1}{a(s)} \int_{t_{1}}^{s} \frac{1}{b(u)} \Delta u \Delta s, \quad B(t)=\int_{\tau(t)}^{\xi(\tau(t))} \frac{1}{a(s)} \int_{s}^{\xi(s)} \frac{1}{b(u)} \Delta u \Delta s, \quad t_{1} \geq t_{0} .
$$

The paper is organized as follows. In Sect. 2, we present some basic definitions and useful results from the theory of calculus on time scales and several lemmas. In Sect. 3, we investigate the oscillation of (1.1) by making a comparison with first-order dynamic equations and establish some sufficient conditions for (1.1) to be oscillatory. In Sect. 4, we give new results for asymptotic behavior of nonoscillatory solutions of (1.1). In Sect. 5, we offer an example to illustrate the feasibility of our primary results.

\section{Preliminaries}

We define the graininess function $\mu$ :

$$
\mu(t)=\sigma(t)-t
$$

and for any function $f: \mathbb{T} \rightarrow \mathbb{R}$ the notation $f^{\sigma}(t)$ denotes $f(\sigma(t))$.

The set of functions $f: \mathbb{T} \rightarrow \mathbb{R}$ that are delta differentiable on $[c, d]_{\mathbb{T}}$ and whose delta derivative function is rd-continuous on $[c, d]_{\mathbb{T}}$ is denoted by $C_{r d}^{1}$.

A function $p: \mathbb{T} \rightarrow \mathbb{R}$ is said to be regressive if

$$
1+\mu(t) p(t) \neq 0, \quad t \in \mathbb{T} .
$$

We denote the set of all $F: \mathbb{T} \rightarrow \mathbb{R}$ which are right-dense continuous and regressive by $\Re$. The exponential function is defined by

$$
e_{p}(t, s)=\exp \left(\int_{s}^{t} \zeta_{\mu(\tau)}(p(\tau)) \Delta \tau\right) \quad \text { for } t \in \mathbb{T}, s \in \mathbb{T},
$$

where $p \in \Re$, the cylinder transformation $\zeta_{h}(z)$ is given by

$$
\zeta_{h}(z)= \begin{cases}\frac{\log (1+h z)}{h} & \text { if } h \neq 0 \\ z & \text { if } h=0 .\end{cases}
$$

For more details, see [3].

Equation (1.1) covers the couple of three-order binomial dynamic equations:

$$
\left[b(t)\left(a(t) x^{\Delta}(t)\right)^{\Delta}\right]^{\Delta}+p(t) f(x(\tau(t)))=0
$$

and

$$
\left[b(t)\left(a(t) x^{\Delta}(t)\right)^{\Delta}\right]^{\Delta}-q(t) h(x(\delta(t)))=0 .
$$

We point out that the solution spaces of (2.2) are different from the solution spaces of (2.3). Let $S$ be the set of all positive solutions of the considered equations. Then for (2.2) the set $S$ is the union of $S_{0}$ and $S_{2}$ :

$$
S=S_{0} \cup S_{2},
$$


where the positive solution sets $S_{0}$ and $S_{2}$ for (2.2) satisfy

$$
\begin{array}{llll}
x(t) \in S_{0} & \Leftrightarrow \quad X(t)=a(t) x^{\Delta}(t)<0, & b(t) X^{\Delta}(t)>0, & {\left[b(t) X^{\Delta}(t)\right]^{\Delta}<0,} \\
x(t) \in S_{2} \quad \Leftrightarrow \quad & \quad X(t)=a(t) x^{\Delta}(t)>0, & b(t) X^{\Delta}(t)>0, & {\left[b(t) X^{\Delta}(t)\right]^{\Delta}<0 .}
\end{array}
$$

Similarly, for (2.3), the set $S$ is also the union of $S_{1}$ and $S_{3}$ :

$$
S=S_{1} \cup S_{3},
$$

where the positive solution sets $S_{1}$ and $S_{3}$ for (2.3) satisfy

$$
\begin{array}{llll}
x(t) \in S_{1} & \Leftrightarrow \quad X(t)=a(t) x^{\Delta}(t)>0, & b(t) X^{\Delta}(t)<0, & {\left[b(t) X^{\Delta}(t)\right]^{\Delta}>0,} \\
x(t) \in S_{3} \quad \Leftrightarrow \quad & X X(t)=a(t) x^{\Delta}(t)>0, & b(t) X^{\Delta}(t)>0, & {\left[b(t) X^{\Delta}(t)\right]^{\Delta}>0 .}
\end{array}
$$

Lemma 2.1 Assume that $x$ is an eventually positive solution of (2.2) or (2.3). Then, for (2.2), there exists $t_{1} \in\left[t_{0}, \infty\right)_{\mathbb{T}}$ such that, for $t \in\left[t_{1}, \infty\right)_{\mathbb{T}}$, either

$$
x(t) \in S_{0} \quad \text { or } \quad x(t) \in S_{2} .
$$

And, for (2.3), we have also either

$$
x(t) \in S_{1} \quad \text { or } \quad x(t) \in S_{3} .
$$

The proof is similar to that of [10, Lemma 1].

Lemma 2.2 Assume that $g \in C_{r d}(\mathbb{T},[0, \infty)), m: \mathbb{T} \rightarrow \mathbb{R}$ is nondecreasing, $n: \mathbb{T} \rightarrow \mathbb{T}$ is nonincreasing, and $j: \mathbb{T} \rightarrow \mathbb{T}$ is nondecreasing. If $s<t$, then

$$
\int_{s}^{\sigma(t)} g(u) m(n(j(u))) \Delta u \geq m(n(j(t))) \int_{s}^{\sigma(t)} g(u) \Delta u .
$$

Proof Since $s<t$, we can divide the integral into two parts:

$$
\int_{s}^{\sigma(t)} g(u) m(n(j(u))) \Delta u=\int_{s}^{t} g(u) m(n(j(u))) \Delta u+\int_{t}^{\sigma(t)} g(u) m(n(j(u))) \Delta u .
$$

Using the fact that $m$ is nondecreasing, $n$ is nonincreasing, and $j$ is nondecreasing, the first part gives

$$
\int_{s}^{t} g(u) m(n(j(u))) \Delta u \geq m\left(n(j(t)) \int_{s}^{t} g(u) \Delta u .\right.
$$

Since $g \circ m \circ n \circ j$ is rd-continuous, by $\int_{t}^{\sigma(t)} y(t) \Delta t=\mu(t) y(t)$, the second part yields

$$
\begin{aligned}
\int_{t}^{\sigma(t)} g(u) m(n(j(u))) \Delta u & =\mu(t) g(t) m(n(j(t))) \\
& =m(n(j(t)))(\mu(t) g(t))=m(n(j(t))) \int_{t}^{\sigma(t)} g(u) \Delta u .
\end{aligned}
$$


Combining (2.4) and (2.5), we get

$$
\begin{aligned}
\int_{s}^{\sigma(t)} g(u) m(n(j(u))) \Delta u & \geq m(n(j(t)))\left(\int_{s}^{t} g(u) \Delta u+\int_{t}^{\sigma(t)} g(u) \Delta u\right) \\
& =m(n(j(t))) \int_{s}^{\sigma(t)} g(u) \Delta u .
\end{aligned}
$$

This completes the proof.

\section{The oscillation of Equation (1.1)}

When we use generalized Riccati transformation techniques, we meet an unexpected obstacle: the nonoscillatory solution space of (1.1) with positive and negative coefficients is unclear from Lemma 2.1. Fortunately, by reducing (1.1) to oscillation of an appropriate first-order delay dynamic equation, we obtain several new oscillatory properties of Equation (1.1) by comparison method.

In this section, we always assume that

(A5) $h$ is bounded, $f$ is nondecreasing, and $f(u v) \geq f(u) f(v)$ for $u v>0$;

(A6) $\int_{t_{0}}^{\infty} \frac{1}{a(t)} \int_{t}^{\infty} \frac{1}{b(s)} \int_{s}^{\infty} q(u) \Delta u \Delta s \Delta t<\infty$.

Hypothesis (A6) reveals that the positive term of (1.1) is dominating. So the structure of the positive solution space of (1.1) is similar to that of (2.2).

Theorem 3.1 Assume that either

$$
\int_{t_{0}}^{\infty} p(u) \Delta u=\infty
$$

or

$$
\int_{t_{0}}^{\infty} p(u) \Delta u<\infty, \quad \int_{t_{0}}^{\infty} \frac{1}{a(v)} \int_{v}^{\infty} \frac{1}{b(s)} \int_{s}^{\infty} p(u) \Delta u \Delta s \Delta v=\infty .
$$

If the first-order nonlinear delay dynamic equation

$$
y^{\Delta}(t)+p(t) f(A(\tau(t))) f(y(\tau(t)))=0
$$

oscillates, then every solution $x$ of $(1.1)$ either is oscillatory or $\lim _{t \rightarrow \infty} x(t)=0$.

Proof Suppose to the contrary that $x(t)$ is an eventually positive solution of (1.1). Then there exists $t_{1} \in \mathbb{T}$ large enough and $t_{1} \geq t_{0}$ such that

$$
x(t)>0, \quad x(\tau(t))>0, \quad x(\delta(t))>0 \quad \text { for all } t \geq t_{1} .
$$

Let

$$
k(t)=x(t)+\int_{t}^{\infty} \frac{1}{a(v)} \int_{v}^{\infty} \frac{1}{b(s)} \int_{s}^{\infty} q(u) h(x(\delta(u))) \Delta u \Delta s \Delta v .
$$

Conditions (A5) and (A6) imply that the function $k(t)$ exists for all $t \geq t_{1}$ and is well defined. Moreover,

$$
k(t)>x(t)>0, \quad k^{\Delta}(t)<x^{\Delta}(t) .
$$


By (3.4) and (1.1), we find that

$$
\left[b(t)\left(a(t) k^{\Delta}(t)\right)^{\Delta}\right]^{\Delta}+p(t) f(x(\tau(t)))=0,
$$

which implies that

$$
\left[b(t)\left(a(t) k^{\Delta}(t)\right)^{\Delta}\right]^{\Delta}=-p(t) f(x(\tau(t)))<0 .
$$

Hence, a suitable modification of Lemma 2.1 together with condition (A2) implies that either

$$
k(t) \in S_{0} \quad \Leftrightarrow \quad a(t) k^{\Delta}(t)<0, \quad b(t)\left(a(t) k^{\Delta}(t)\right)^{\Delta}>0, \quad\left[b(t)\left(a(t) k^{\Delta}(t)\right)^{\Delta}\right]^{\Delta}<0,
$$

or

$$
k(t) \in S_{2} \quad \Leftrightarrow \quad a(t) k^{\Delta}(t)>0, \quad b(t)\left(a(t) k^{\Delta}(t)\right)^{\Delta}>0, \quad\left[b(t)\left(a(t) k^{\Delta}(t)\right)^{\Delta}\right]^{\Delta}<0,
$$

for all $t \geq t_{1}$. Firstly, we consider the case $k(t) \in S_{0}$. Since $k(t)>0$ and $k^{\Delta}(t)<0$, by (3.4), we find

$$
\lim _{t \rightarrow \infty} k(t)=\lim _{t \rightarrow \infty} x(t)=2 l \geq 0 .
$$

We claim that $l=0$. Otherwise, $l>0$, then $x(\tau(t)) \geq l>0$ holds for all $t \geq t_{1}$.

If (3.1) holds, noting that $f$ is nondecreasing, integrate $(3.5)$ from $t_{1}$ to $t\left(t \in\left[t_{1}, \infty\right)_{\mathbb{T}}\right)$

$$
b(t)\left(a(t) k^{\Delta}(t)\right)^{\Delta} \leq b\left(t_{1}\right)\left(a\left(t_{1}\right) k^{\Delta}\left(t_{1}\right)\right)^{\Delta}-f(l) \int_{t_{1}}^{t} p(s) \Delta s \rightarrow-\infty, \quad t \rightarrow \infty,
$$

which contradicts $b(t)\left(a(t) k^{\Delta}(t)\right)^{\Delta}>0$.

On the other hand, if (3.2) holds, similar to the proof of [7, Theorem 2.1], we find the limit $\lim _{t \rightarrow \infty} k(t)=-\infty$, which contradicts the fact $k(t)>0$. So,

$$
\lim _{t \rightarrow \infty} k(t)=0 \quad \text { and } \quad \lim _{t \rightarrow \infty} x(t)=0 .
$$

Now we consider $k(t) \in S_{2}$. By $\left[b(t)\left(a(t) k^{\Delta}(t)\right)^{\Delta}\right]^{\Delta}<0$, we get two inequalities:

$$
\begin{aligned}
a(t) k^{\Delta}(t) & \geq a(t) k^{\Delta}(t)-a\left(t_{1}\right) k^{\Delta}\left(t_{1}\right) \\
& =\int_{t_{1}}^{t} b(s)\left(a(s) k^{\Delta}(s)\right)^{\Delta} \frac{1}{b(s)} \Delta s \\
& \geq b(t)\left(a(t) k^{\Delta}(t)\right)^{\Delta} \int_{t_{1}}^{t} \frac{1}{b(s)} \Delta s
\end{aligned}
$$

and

$$
k^{\Delta}(t) \geq \frac{b(t)}{a(t)}\left(a(t) k^{\Delta}(t)\right)^{\Delta} \int_{t_{1}}^{t} \frac{1}{b(s)} \Delta s
$$


The last inequality together with $x(t)>0$ and $k^{\Delta}(t)<x^{\Delta}(t)$ shows that

$$
\begin{aligned}
x(t) & \geq \int_{t_{1}}^{t} x^{\Delta}(s) \Delta s \geq \int_{t_{1}}^{t} k^{\Delta}(s) \Delta s \\
& \geq \int_{t_{1}}^{t} \frac{b(s)}{a(s)}\left(a(s) k^{\Delta}(s)\right)^{\Delta} \int_{t_{1}}^{s} \frac{1}{b(u)} \Delta u \Delta s \\
& \geq b(t)\left(a(t) k^{\Delta}(t)\right)^{\Delta} \int_{t_{1}}^{t} \frac{1}{a(s)} \int_{t_{1}}^{s} \frac{1}{b(u)} \Delta u \Delta s .
\end{aligned}
$$

Let

$$
z_{1}(t)=b(t)\left(a(t) k^{\Delta}(t)\right)^{\Delta}
$$

By (3.6) and $z_{1}(t)=b(t)\left(a(t) k^{\Delta}(t)\right)^{\Delta}>0$, we have

$$
x(t) \geq z_{1}(t) \int_{t_{1}}^{t} \frac{1}{a(s)} \int_{t_{1}}^{s} \frac{1}{b(u)} \Delta u \Delta s>0 .
$$

According to (3.5), (A5), and the definition of $A(t)$, we see that $z_{1}>0$ is a solution of the delay dynamic inequality

$$
y^{\Delta}(t)+p(t) f(A(\tau(t))) f(y(\tau(t))) \leq 0 .
$$

By [4, Theorem 3.1], it follows that the corresponding first-order delay dynamic equation (3.3) has a positive solution, too. This contradiction shows that $k(t) \notin S_{2}$. This completes the proof.

Remark 3.2 Note that (A2) implies that

$$
\lim _{t \rightarrow \infty} A(t)=\lim _{t \rightarrow \infty} \int_{t_{1}}^{t} \frac{1}{a(s)} \int_{t_{1}}^{s} \frac{1}{b(u)} \Delta u \Delta s=\infty
$$

Then there exists $t_{2} \geq t_{1}$ such that, for $t \geq t_{2}>t_{1}$,

$$
\begin{aligned}
& \int_{t_{1}}^{t_{2}} \frac{1}{a(s)} \int_{t_{1}}^{s} \frac{1}{b(u)} \Delta u \Delta s \geq 1, \\
& x(t) \geq z_{1}(t) \int_{t_{1}}^{t} \frac{1}{a(s)} \int_{t_{1}}^{s} \frac{1}{b(u)} \Delta u \Delta s \geq z_{1}(t) \int_{t_{1}}^{t_{2}} \frac{1}{a(s)} \int_{t_{1}}^{s} \frac{1}{b(u)} \Delta u \Delta s \geq z_{1}(t) .
\end{aligned}
$$

Set it into (3.5),

$$
y^{\Delta}(t)+p(t) f(y(\tau(t))) \leq 0
$$

Thus, we can remove the condition $f(u v) \geq f(u) f(v)$ for $u v>0$ in (A5) and (3.3) can be replaced by

$$
y^{\Delta}(t)+p(t) f(y(\tau(t)))=0
$$


to make sure that the conclusion of Theorem 3.1 is also true. However, [7, Theorem 2.1] did not give the corresponding result. When we use oscillation criterion (3.9) of Corollary 3.3, we also need not the condition $f(u v) \geq f(u) f(v)$ for $u v>0$ in (A5).

Corollary 3.3 Let (3.1) or (3.2) hold. If

$$
\limsup _{t \rightarrow \infty} \int_{\tau(t)}^{\sigma(t)} p(s) f(A(\tau(s))) \Delta s>\limsup _{u \rightarrow 0} \frac{u}{f(u)}
$$

or

$$
\limsup _{t \rightarrow \infty} \int_{\tau(t)}^{\sigma(t)} p(s) \Delta s>\limsup _{u \rightarrow 0} \frac{u}{f(u)}
$$

then every solution $x$ of $(1.1)$ either oscillates or $\lim _{t \rightarrow \infty} x(t)=0$.

Proof Due to Theorem 3.1 and 3.2, we only need to show that (3.3) and (3.7) oscillate.

First, assume that Equation (3.3) has a positive solution $z(t)$. Since $\lim _{t \rightarrow \infty} \tau(t)=\infty$, there is a positive number $t_{1} \geq t_{0}$ such that $z(\tau(t))>0$ for $t \geq t_{1}$. By (3.3),

$$
z^{\Delta}(t)=-p(t) f(A(\tau(t))) f(z(\tau(t)))<0, \quad t \geq t_{1},
$$

which implies the limit $\lim _{t \rightarrow \infty} z(t)=r \geq 0$.

Integrating (3.10) from $\tau(t)$ to $\sigma(t)$ yields

$$
z(\sigma(t))-z(\tau(t))+\int_{\tau(t)}^{\sigma(t)} p(s) f(A(\tau(s))) f(z(\tau(s))) \Delta s=0 .
$$

Since $z$ is $\Delta$-differentiable and rd-continuous and the function $\tau$ is nondecreasing, Lemma 2.2 is applicable for the integral term in the previous equation. By Lemma 2.2, we get

$$
\int_{\tau(t)}^{\sigma(t)} p(s) f(A(\tau(s))) f(z(\tau(s))) \Delta s \geq f(z(\tau(t))) \int_{\tau(t)}^{\sigma(t)} p(s) f(A(\tau(s))) \Delta s,
$$

So

$$
z(\tau(t)) \geq z(\sigma(t))+f(z(\tau(t))) \int_{\tau(t)}^{\sigma(t)} p(s) f(A(\tau(s))) \Delta s .
$$

Note that $z(t)>0, p(t)>0, z f(z)>0$ for all $t \geq t_{1}$, we have

$$
\frac{z(\tau(t))}{f(z(\tau(t)))} \geq \int_{\tau(t)}^{\sigma(t)} p(s) f(A(\tau(s))) \Delta s, \quad t \geq t_{1}
$$

Taking upper limits on both sides brings an obvious contradiction with (3.8).

Second, assume that Equation (3.7) has a positive solution $z_{2}(t)$. The proof is the same as the previous case and we omit it.

Let $f(u)=|u|^{\beta-1} u$. We have the following result. 
Corollary 3.4 Let $0<\beta<1$. Assume that (3.1) or (3.2) holds and

$$
\limsup _{t \rightarrow \infty} \int_{\tau(t)}^{\sigma(t)} p(s) A^{\beta}(\tau(s)) \Delta s>0
$$

then every solution $x$ of the nonlinear dynamic equation

$$
\left[b(t)\left(a(t) x^{\Delta}(t)\right)^{\Delta}\right]^{\Delta}+p(t)|x(\tau(t))|^{\beta-1} x(\tau(t))-q(t) h(x(\delta(t)))=0
$$

either oscillates or $\lim _{t \rightarrow \infty} x(t)=0$.

Remark 3.5 When (3.2) holds, if (3.11) is replaced by

$$
\limsup _{t \rightarrow \infty} \int_{\tau(t)}^{\sigma(t)} p(s) \Delta s>0
$$

the conclusion of Corollary 3.4 is also true. What is more, if $\beta=1$

$$
\limsup _{t \rightarrow \infty} \int_{\tau(t)}^{\sigma(t)} p(s) A(\tau(s)) \Delta s>1
$$

then the conclusion of Corollary 3.4 still holds.

Compared with the following Corollary 3.6, we can see that (3.15) is better than (3.14). But it is easier to compute (3.14) than (3.15). When $\mathbb{T}=\mathbb{R},[22$, Example 2] shows that [7, Corollary 2.2] is a special case of Corollary 3.6.

Corollary 3.6 Let (3.1) or (3.2) hold. If

$$
\limsup _{t \rightarrow \infty} \sup _{\lambda \in E}\left\{\lambda \exp _{-\lambda p(A \circ \tau)}(\tau(t), t)\right\}<1,
$$

where $E=\{\lambda \mid \lambda>0,1-\lambda p(t) A(\tau(t)) \mu(t)>0, t \in \mathbb{T}\}$, then every solution $x$ of the delay $d y$ namic equation with positive and negative coefficients

$$
\left[b(t)\left(a(t) x^{\Delta}(t)\right)^{\Delta}\right]^{\Delta}+p(t) x(\tau(t))-q(t) h(x(\delta(t)))=0
$$

either oscillates or $\lim _{t \rightarrow \infty} x(t)=0$.

Proof A modification of [22, Corollary 2] guarantees (3.3) to be oscillatory with $f(u)=u$. The conclusion of Corollary 3.6 is correct owing to Theorem 3.1.

On the other hand, using the supplementary condition, we will establish another oscillation criterion for (1.1) to make sure that (1.1) is oscillatory.

Theorem 3.7 Assume that there exists a function $\xi \in C_{r d}^{1}\left(\left[t_{0}, \infty\right)_{\mathbb{T}}, \mathbb{R}\right)$ such that

$$
\xi^{\Delta}(t) \geq 0, \quad \xi(t)>t, \quad \eta(t):=\xi(\xi(\tau(t)))<t .
$$


If both the first-order delay dynamic equation (3.3) and

$$
y^{\Delta}(t)+p(t) f(B(t)) f(y(\eta(t)))=0
$$

are oscillatory, then (1.1) is oscillatory.

Proof Assume not. Let $x$ be an eventually positive solution of (1.1). The function $k(t)$ is defined by (3.4). As in the proof of Theorem 3.1, we see that $k(t) \in S_{0}$, and there exists $\lim _{t \rightarrow \infty} k(t)=2 l \geq 0$. Assumption (A2) implies

$$
\lim _{t \rightarrow \infty} b(t)\left(a(t) k^{\Delta}(t)\right)^{\Delta}=\lim _{t \rightarrow \infty} a(t) k^{\Delta}(t)=0 .
$$

In view of (3.4), it follows that

$$
\lim _{t \rightarrow \infty} x(t)=2 l, \quad \lim _{t \rightarrow \infty} b(t)\left(a(t) x^{\Delta}(t)\right)^{\Delta}=\lim _{t \rightarrow \infty} a(t) x^{\Delta}(t)=0 .
$$

Let

$$
w(t)=x(t)+\int_{t}^{\infty} \frac{1}{a(v)} \int_{t_{1}}^{v} \frac{1}{b(s)} \int_{t_{1}}^{s} q(u) h(x(\delta(u))) \Delta u \Delta s \Delta v .
$$

Conditions (A5) and (A6) imply that $w(t)$ is well defined. By (A1), (A3), (1.1), and (3.20), we have

$$
w(t)>x(t)>0, \quad a(t) w^{\Delta}(t)<a(t) x^{\Delta}(t), \quad b(t)\left(a(t) w^{\Delta}(t)\right)^{\Delta}<b(t)\left(a(t) x^{\Delta}(t)\right)^{\Delta}
$$

and

$$
\left[b(t)\left(a(t) w^{\Delta}(t)\right)^{\Delta}\right]^{\Delta}=-p(t) f(x(\tau(t)))<0 .
$$

Then, hypothesis (A2) combined with a proper modification of Lemma 2.1 implies that either

$$
w \in S_{0} \quad \Leftrightarrow \quad w>0, \quad a w^{\Delta}<0, \quad b\left(a w^{\Delta}\right)^{\Delta}>0, \quad\left[b\left(a w^{\Delta}\right)^{\Delta}\right]^{\Delta}<0
$$

or

$$
w \in S_{2} \quad \Leftrightarrow \quad w>0, \quad a w^{\Delta}>0, \quad b\left(a w^{\Delta}\right)^{\Delta}>0, \quad\left[b\left(a w^{\Delta}\right)^{\Delta}\right]^{\Delta}<0,
$$

eventually. However, $w(t) \in S_{2}$ will not happen. In fact, if $w(t) \in S_{2}$, then by $0<a(t) w^{\Delta}(t)<$ $a(t) x^{\Delta}(t)$ and $\lim _{t \rightarrow \infty} a(t) x^{\Delta}(t)=0$, we get $\lim _{t \rightarrow \infty} a(t) w^{\Delta}(t)=0$. We have a contradiction with $a(t) w^{\Delta}(t)>0$ and $\left(a(t) w^{\Delta}(t)\right)^{\Delta}>0$, eventually. So, we claim that $w(t) \in S_{0}$.

Let $\psi(t)=b(t)\left(a(t) w^{\Delta}(t)\right)^{\Delta}$, then $\psi^{\Delta}(t)=\left(b(t)\left(a(t) w^{\Delta}(t)\right)^{\Delta}\right)^{\Delta}<0$. By (3.17) and (3.19), integrate $\left(a(t) w^{\Delta}(t)\right)^{\Delta}<\left(a(t) x^{\Delta}(t)\right)^{\Delta}$ from $t$ to $\infty$,

$$
\begin{aligned}
-a(t) x^{\Delta}(t) & \geq \int_{t}^{\infty} \frac{b(s)\left(a(s) w^{\Delta}(s)\right)^{\Delta}}{b(s)} \Delta s \\
& \geq \int_{t}^{\xi(t)} \frac{b(s)\left(a(s) w^{\Delta}(s)\right)^{\Delta}}{b(s)} \Delta s \geq \psi(\xi(t)) \int_{t}^{\xi(t)} \frac{1}{b(s)} \Delta s
\end{aligned}
$$


which implies $-x^{\Delta}(t) \geq \psi(\xi(t)) \frac{1}{a(t)} \int_{t}^{\xi(t)} \frac{1}{b(s)} \Delta s$. We carry out the calculation

$$
x(\tau(t)) \geq x(\tau(t))-x(\xi(\tau(t))) \geq \int_{\tau(t)}^{\xi(\tau(t))} \frac{\psi(\xi(u))}{a(u)} \int_{u}^{\xi(u)} \frac{1}{b(s)} \Delta s \Delta u \geq \psi(\eta(t)) B(t) .
$$

Substituting it into (3.21), we find that the delay dynamic inequality

$$
y^{\Delta}(t)+p(t) f(B(t)) f(y(\eta(t))) \leq 0
$$

has a solution $\psi>0$. Owing to [4, Theorem 3.1], the corresponding first-order dynamic equation (3.18) has a positive solution as well. The contradiction shows that $w(t) \notin S_{0}$ and we reach an agreement that (1.1) oscillates.

It is evident that Corollaries 3.8, 3.9, 3.10 are true.

Corollary 3.8 Assume that (3.8) and (3.17) hold.If

$$
\limsup _{t \rightarrow \infty} \int_{\tau(t)}^{\sigma(t)} p(s) f(B(s)) \Delta s>\limsup _{u \rightarrow 0} \frac{u}{f(u)}
$$

then (1.1) oscillates.

Corollary 3.9 Assume that (3.15) and (3.17) hold. If

$$
\limsup _{t \rightarrow \infty} \sup _{\lambda \in E^{\prime}}\left\{\lambda \exp _{-\lambda p B}(\tau(t), t)\right\}<1,
$$

where $E^{\prime}=\{\lambda \mid \lambda>0,1-\lambda p(t) B(t) \mu(t)>0, t \in \mathbb{T}\}$, then (3.16) oscillates.

Corollary 3.10 Assume that (3.11) and (3.17) hold.If

$$
\limsup _{t \rightarrow \infty} \int_{\tau(t)}^{\sigma(t)} p(s) B^{\beta}(s) \Delta s>0
$$

then (3.12) oscillates.

Remark 3.11 When $\mathbb{T}=\mathbb{R}$, Theorem 3.7 and Corollaries 3.8, 3.9, 3.10 reduce to Theorem 2.6 and Corollaries 2.8-2.10 in [7].

\section{The asymptotic properties of Equation (1.1)}

Now, we explore the asymptotic behavior of solutions for (1.1) by reducing the exploration of (1.1) to oscillation of a proper first-order dynamic equation.

In this section, we need the following set of hypotheses.

(A7) $f$ is bounded, $h$ is nondecreasing;

(A8) $\int_{t_{0}}^{\infty} \frac{1}{a(t)} \int_{t}^{\infty} \frac{1}{b(s)} \int_{s}^{\infty} p(u) \Delta u \Delta s \Delta t<\infty$.

Theorem 4.1 Suppose that

$$
\int_{t_{0}}^{\infty} q(u)|h( \pm c A(\delta(u)))| \Delta u=\infty
$$


holds for every $c>0$. If the first-order nonlinear delay dynamic equation

$$
y^{\Delta}(t)-\left[\frac{1}{a(t)} \int_{t}^{\infty} \frac{1}{b(u)} \int_{u}^{\infty} q(s) \Delta s \Delta u\right] h(y(\delta(t)))=0
$$

is oscillatory, then every nonoscillatory solution $x$ of (1.1) either

$$
\lim _{t \rightarrow \infty} \frac{|x(t)|}{A(t)}=\infty
$$

or, for some $M>0$,

$$
|x(t)| \leq M \int_{t}^{\infty} \frac{1}{a(v)} \int_{v}^{\infty} \frac{1}{b(s)} \int_{s}^{\infty} p(u) \Delta u \Delta s \Delta v, \quad \lim _{t \rightarrow \infty} x(t)=0 .
$$

Proof Suppose to contrary that (1.1) has a nonoscillatory solution $x>0$. We may assume that there exists $t_{1} \geq t_{0}$ such that

$$
x(t)>0, \quad x(\tau(t))>0, \quad x(\delta(t))>0 \quad \text { for all } t \geq t_{1} .
$$

Let

$$
r(t)=x(t)-\int_{t}^{\infty} \frac{1}{a(v)} \int_{v}^{\infty} \frac{1}{b(s)} \int_{s}^{\infty} p(u) f(x(\tau(u))) \Delta u \Delta s \Delta v .
$$

According to (A7) and (A8), $r(t)$ exists for all $t \in \mathbb{T}$ and is well defined. Furthermore, $r(t)<$ $x(t)$, and

$$
\left[b(t)\left(a(t) r^{\Delta}(t)\right)^{\Delta}\right]^{\Delta}=q(t) h(x(\delta(t)))>0 .
$$

Hence, by (A2), a proper modification of Lemma 2.1 implies that, for all $t \geq t_{1}$, either

$$
r \in S_{1} \quad \Leftrightarrow \quad r>0, \quad a r^{\Delta}>0, \quad b\left(a r^{\Delta}\right)^{\Delta}<0, \quad\left[b\left(a r^{\Delta}\right)^{\Delta}\right]^{\Delta}>0
$$

or

$$
r \in S_{3} \quad \Leftrightarrow \quad r>0, \quad a r^{\Delta}>0, \quad b\left(a r^{\Delta}\right)^{\Delta}>0, \quad\left[b\left(a r^{\Delta}\right)^{\Delta}\right]^{\Delta}>0,
$$

or

$$
r(t)<0 \text {. }
$$

The rest of the proof is similar to that of [2, Theorem 1].

The function $r \in S_{3}$ implies

$$
b(t)\left(a(t) r^{\Delta}(t)\right)^{\Delta} \geq c=b\left(t_{1}\right)\left(a\left(t_{1}\right) r^{\Delta}\left(t_{1}\right)\right)^{\Delta} .
$$


By direct calculation, we have

$$
x(t) \geq r(t) \geq c \int_{t_{1}}^{t} \frac{1}{a(s)} \int_{t_{1}}^{s} \frac{1}{b(u)} \Delta u \Delta s=c A(t) \rightarrow \infty \quad \text { as } t \rightarrow \infty .
$$

Hence, if we remove condition (4.1), we conclude that every nonoscillatory solution $x$ of (1.1) either is unbounded or converges to zero. The following result improves [2, Theorem 1].

Remark 4.2 If (4.2) is oscillatory, then every nonoscillatory solution $x$ of (1.1) either, for sufficiently large $T \in \mathbb{T}$ and some $c>0$, is such that

$$
|x(t)| \geq c A(t) \quad \text { for } t \geq T \quad \text { and } \quad \lim _{t \rightarrow \infty}|x(t)|=\infty,
$$

or satisfies (4.4).

Furthermore, applying extra condition, we obtain the other new criterion that every nonoscillatory solution of (1.1) is asymptotic stable.

Theorem 4.3 Assume that there exists a function $\chi \in C_{r d}^{1}\left(\left[t_{0}, \infty\right)_{\mathbb{T}}, \mathbb{R}\right)$ such that

$$
\chi^{\Delta}(t) \geq 0, \quad \chi(t)<t, \quad \varrho(t):=\delta(\chi(\chi(t)))>t .
$$

If both the first-order delay dynamic equation (4.2) and

$$
\omega^{\Delta}(t)-\left[\frac{1}{a(t)} \int_{\chi(t)}^{t} \frac{1}{b(u)} \int_{\chi(u)}^{u} q(s) \Delta s \Delta u\right] h(\omega(\varrho(t))=0
$$

are oscillatory, then every nonoscillatory solution of (1.1) satisfies (4.4).

Proof The proof is similar to [2, Theorem 2].

Remark 4.4 When $\mathbb{T}=\mathbb{R}$, Theorems 4.1 and 4.3 reduce to Theorems $1-2$ in [2].

\section{Application}

In this section, we present an example to illustrate the feasibility of our results.

Example 5.1 Consider the following third-order delay dynamic equation with positive and negative coefficients:

$$
\left[b(t)\left(a(t) x^{\Delta}(t)\right)^{\Delta}\right]^{\Delta}+p(t) x(\tau(t))-q(t) \frac{x(\delta(t)}{2+|x(\delta(t))|^{3}}=0
$$

for $t \in\left[t_{0}, \infty\right)_{\mathbb{T}}$, where $b, a, p, q \in C_{r d}\left([1, \infty)_{\mathbb{T}}, \mathbb{R}^{+}\right), \tau(t) \leq t, \delta(t) \geq t, \lim _{t \rightarrow \infty} \tau(t)=\infty$. Now $f(u)=u, h(u)=\frac{x(\delta(t))}{2+|x(\delta(t))|^{3}}$ is bounded, so conditions (A1), (A3)-(A4) are satisfied. Then we can choose $a, b$ satisfying (A2) and choose $p, q$ satisfying (A6) and (3.1).

(I) $\mathbb{T}=\mathbb{R}$. Taking account of Corollary 3.6, (3.15) can be written as follows:

$$
\limsup _{t \rightarrow \infty} \sup _{\lambda \in E}\left\{\lambda \int_{\tau(t)}^{t}(-\lambda p(s) A(\tau(s)) d s)\right\}<1,
$$


where $E=\mathbb{R}^{+}$. Let

$$
f(\lambda)=\lambda \exp \left\{\int_{\tau(t)}^{t}(-\lambda p(s) A(\tau(s)) d s\}\right.
$$

It follows from Lagrange theorem that

$$
\sup _{\lambda \in E} f(\lambda)=\frac{1}{e \int_{\tau(t)}^{t} p(s) A(\tau(s)) d s}<1
$$

Therefore, if

$$
\liminf _{t \rightarrow \infty} \int_{\tau(t)}^{t} p(s) A(\tau(s)) d s>\frac{1}{e}
$$

then the differential equation

$$
y^{\prime}(t)+p(t) A(\tau(t)) y(\tau(t))=0
$$

is oscillatory. That is just a modification of [18, Theorem 1].

(II) $\mathbb{T}=h \mathbb{Z}=\{h k \mid k \in \mathbb{Z}\}$ for $h>0$. Assume that $p(t) A(\tau(t))=1, \tau(t)=t-l h$ with $l \in Z^{+}$, and from (3.15) we get

$$
\sup _{\lambda \in E}\left\{\lambda(1-h \lambda)^{k}\right\}<1
$$

where $E=\left\{\lambda \mid 0<\lambda<\frac{1}{h}\right\}$. By simply calculating, (5.4) turns out to be

$$
\frac{l^{l}}{(k+1)^{k+1}}<h .
$$

So, if we pick up some $h>0$ such that (5.5) holds, then the equation

$$
y^{\Delta}(t)+y(\tau(t))=0
$$

oscillates.

If we apply Remark 3.5 ,

$$
\int_{\tau(t)}^{\sigma(t)} p(s) A(\tau(s)) \Delta s=\sigma(t)-\tau(t)=t+h-(t-l h)=(1+l) h>1
$$

implies that $\frac{1}{k+1}<h$ guarantees Equation (5.6) to oscillate. Note that

$$
\frac{l^{l}}{(k+1)^{k+1}}<\frac{1}{k+1}<h,
$$

this reveals that (3.15) is better than (3.14). However, it is convenient to compute (3.14), but not to compute (3.15). 
(III) $\mathbb{T}=\left\{t_{n}=\sum_{k=1}^{n} \frac{1}{k}: n \in \mathbb{N}\right\}$. Assume that $p(t) A(\tau(t))=\alpha$, where $\alpha$ is an integer. By (3.15), we see that, if

$$
\lim _{k \rightarrow \infty} \sup _{n>k} \sup _{\lambda \in E}\left\{\lambda C_{n-\lambda m}^{n-\lambda m+\alpha}\right\} d s<1,
$$

where $E=\left\{\lambda \mid 0<\lambda<\frac{n}{\alpha}\right\}$, then the equation

$$
y^{\Delta}\left(\sum_{k=1}^{n} \frac{1}{k}\right)+\alpha y\left(\sum_{k=1}^{n-m} \frac{1}{k}\right)=0
$$

is oscillatory.

By Corollary 3.6, on time scales $\mathbb{T}=\mathbb{R}, \mathbb{T}=h \mathbb{Z}=\{h k \mid k \in \mathbb{Z}\}$ for $h>0$, and $\mathbb{T}=$ $\left\{\sum_{k=1}^{n} \frac{1}{k}, k \in \mathbb{N}\right\}$, then every solution $x$ of (5.1) oscillates or

$$
\lim _{t \rightarrow \infty} x(t)=0
$$

\section{Acknowledgements}

The authors would like to thank Professors Yu Huang and Qi-Ru Wang for their helpful discussions.

\section{Funding}

This project is partially supported by the NSFC of China (Grant Nos. 11271379, 11671406, 11801092, 11801093),

Construction Project of Teaching Quality and Teaching Reform in Guangdong Undergraduate Colleges and Universities in 2018 (Speciality of Financial Mathematics), the Distinctive Innovation of Ordinary University of Guangdong Province (Grant No. 2016KTSCX093), the Funds of Guangzhou Science and Technology (Grant No. 201804010088), and the NSF of Guangdong University of Education (Grant Nos. 2016sjjxjd04, 2018sfzy01).

\section{Competing interests}

The authors declare that they have no competing interests.

\section{Authors' contributions}

All authors contributed equally to this work. All authors read and approved the final manuscript.

\section{Authors' information}

Xianyong Huang, Associate Professor, the major field of interest is in the area of ordinary differential equations and dynamical systems.

\section{Author details}

'Department of Mathematics, Guangdong University of Education, Guangzhou, P.R. China. ${ }^{2}$ Department of Mathematics, College of Medical Information Engineering, Guangdong Pharmaceutical University, Guangzhou, P.R. China.

\section{Publisher's Note}

Springer Nature remains neutral with regard to jurisdictional claims in published maps and institutional affiliations.

Received: 26 February 2019 Accepted: 12 June 2019 Published online: 17 July 2019

\section{References}

1. Agarwal, R.P., O'Regan, D., Saker, S.H.: Oscillation criteria for second-order nonlinear neutral delay dynamic equations. J. Math. Anal. Appl. 300, 203-217 (2004)

2. Baculíková, B., Džrina, J.: Oscillation of functional trinomial differential equations with positive and negative term. Appl. Math. Comput. 295, 47-52 (2017)

3. Bohner, M., Peterson, A.: Dynamic Equations on Time Scales: An Introduction with Applications. Birkhäuser, Boston (2001)

4. Braverman, E., Karpuz, B.: Nonoscillation of first-order dynamic equations with several delays. Adv. Differ. Equ. 2010, Article ID 873459 (2010)

5. Deng, X.H., Wang, Q.R., Agarwal, R.P.: Oscillation and nonoscillation for second order neutral dynamic equations with positive and negative coefficients on time scales. Adv. Differ. Equ. 2014, 115 (2014)

6. Deng, X.H., Wang, Q.R., Zhou, Z:: Oscillation criteria for second order neutral dynamic equations of Emden-Fowler type with positive and negative coefficients on time scales. Sci. China Math. 60, 113-132 (2017)

7. Džrina, J., Baculíková, B.: Oscillation of trinomial differential equations with positive and negative terms. Electron. J. Qual. Theory Differ. Equ. 2014, 43 (2014) 
8. El-Morshedy, H.A.: New oscillation criteria for second order linear difference equations with positive and negative coefficients. Comput. Math. Appl. 58, 1988-1997 (2009)

9. Erbe, L., Mert, R., Peterson, A., Zafer, A.: Oscillation of even order nonlinear delay dynamic equations on time scales. Czechoslov. Math. J. 63, 265-279 (2013)

10. Erbe, L., Peterson, A., Saker, S.H.: Asymptotic behavior of solutions of a third-order nonlinear dynamic equation on time scales. J. Comput. Appl. Math. 181, 92-102 (2005)

11. Erbe, L., Peterson, A., Saker, S.H.: Oscillation and asymptotic behavior a third-order nonlinear dynamic equation. Can. Appl. Math. Q. 14, 129-147 (2006)

12. Erbe, L., Peterson, A., Saker, S.H.: Hille and Nehari type criteria for third-order dynamic equations. J. Math. Anal. Appl. 329, 112-131 (2007)

13. Grace, S.R., Agarwal, R.P., Zafer, A.: Oscillation of higher order nonlinear dynamic equations on time scales. Adv. Differ. Equ. 2012, 67 (2012)

14. Han, Z., Li, T., Sun, S.: Oscillation behavior of solutions of third-order nonlinear delay dynamic equations on time scales. Commun. Korean Math. Soc. 26, 499-513 (2011)

15. Hilger, S.: Analysis on measure chains-a unified approach to continuous and discrete calculus. Results Math. 18 18-56 (1990)

16. Karpuz, B.: Existence and uniqueness of solutions to systems of delay dynamic equations on time scales. Int. J. Math. Comput. 10, 48-58 (2011)

17. Karpuz, B.: Necessary and sufficient conditions on the asymptotic behavior of second-order neutral delay dynamic equations with positive and negative coefficients. Math. Methods Appl. Sci. 37, 1219-1231 (2014)

18. Ladde, G.S., Lakshmikantham, V., Zhang, B.G.: Oscillation Theory of Differential Equations with Deviating Arguments. Dekker, New York (1987)

19. Manojlovic, J., Shoukaku, Y., Tanigawa, T., Yoshida, N.: Oscillation criteria for second order differential equations with positive and negative coefficients. Appl. Math. Comput. 181, 853-863 (2006)

20. Padhi, S.: Oscillation and asymptotic behaviour of solutions of second order homogeneous neutral differential equations with positive and negative coefficients. Funct. Differ. Equ. 14, 363-371 (2007)

21. Padhi, S.: Oscillation and asymptotic behaviour of solutions of second order neutral differential equations with positive and negative coefficients. Fasc. Math. 38, 105-114 (2007)

22. Zhang, B.G., Deng, X.: Oscillation of delay differential equations on time scales. Math. Comput. Model. 36, 1307-1318 (2002)

\section{Submit your manuscript to a SpringerOpen ${ }^{\circ}$ journal and benefit from:}

- Convenient online submission

- Rigorous peer review

- Open access: articles freely available online

- High visibility within the field

Retaining the copyright to your article

Submit your next manuscript at $\boldsymbol{~ s p r i n g e r o p e n . c o m ~}$ 